\title{
Hyperbolic automorphisms of free groups
}

\author{
Peter Brinkmann
}

\begin{abstract}
We prove that an automorphism $\phi: F \rightarrow F$ of a finitely generated free group $F$ is hyperbolic in the sense of Gromov if it has no nontrivial periodic conjugacy classes. This result was previously claimed (but not proved) in [BF92].
\end{abstract}

\section{Introduction}

Let $F$ be a finitely generated free group. We fix a basis once and for all, and denote by $|$.$| the word length with respect to this basis. An automorphism$ $\phi: F \rightarrow F$ is said to be hyperbolic in the sense of Gromov (or just hyperbolic) if there exist numbers $M>0$ and $\lambda>1$ such that

$$
\lambda|g| \leq \max \left\{\left|\phi^{M}(g)\right|,\left|\phi^{-M}(g)\right|\right\}
$$

for all $g \in F$.

An automorphism $\phi: F \rightarrow F$ is called atoroidal if it has no nontrivial periodic conjugacy classes. This definition is motivated by the fact that the mapping torus $F \rtimes_{\phi} \mathbb{Z}$ of such an automorphism contains no subgroups isomorphic to $\mathbb{Z} \oplus \mathbb{Z}$.

The following theorem is the main result of this paper. It was previously claimed in BF92, and a proof in the special case of irreducible automorphisms appeared in BFH97b], I but the general question remained open until now.

Theorem 1.1. If $\phi: F \rightarrow F$ is an atoroidal automorphism, then $\phi$ is hyperbolic.

\footnotetext{
${ }^{1}$ In $[$ BFH97a], the authors state that the main results of [BFH97b] are only proved for irreducible automorphisms with irreducible powers, but a close inspection of their proofs shows that this additional hypothesis is not necessary for the results quoted in this paper.
} 
Theorem 1.1 completes the proof of the following theorem.

Theorem 1.2. Let $\phi$ be an automorphism of F. Then the following statements are equivalent:

1. The mapping torus $F \rtimes_{\phi} \mathbb{Z}$ of $\phi$ is hyperbolic.

2. $\phi$ is hyperbolic.

3. $\phi$ is atoroidal.

The equivalence between (11) and (2) was established in BF92, BF96 and Ger98. (2) $\Rightarrow$ (3) follows immediately from the definitions, and (3) $\Rightarrow$ (2) is precisely our Theorem 1.1.

The train track techniques developed in [BH92, BFH98] will be our most important tool, and in Section 3, we review the results needed in this paper. The key feature of our proof of Theorem 1.1 is that we study the growth of the length of paths under iterates of $\phi^{ \pm 1}$ by analyzing only one train track map representing some iterate of $\phi$, rather than two train track maps representing $\phi$ and $\phi^{-1}$. This was originally suggested by Martin Lustig, and it simplifies the approach considerably as the relationship between train track maps representing $\phi$ and train track maps representing $\phi^{-1}$ remains somewhat mysterious.

I would like to thank Mladen Bestvina, Steve Gersten and Martin Lustig for many helpful discussions. I am indebted to the referee for several helpful suggestions, and I would also like to express my gratitude to Sean SatherWagstaff.

\section{Hyperbolic automorphisms and homotopy equivalences of graphs}

In this section, we list some basic lemmas that will allow us to use train track techniques in the proof of Theorem 1.1. We also introduce some notation that will be useful later.

Lemma 2.1 ([BFH97b]). Let $\phi: F \rightarrow F$ be an atoroidal automorphism. Then $\phi$ is hyperbolic if there exist numbers $M>0$ and $\lambda>1$ such that

$$
\lambda\|g\| \leq \max \left\{\left\|\phi^{M}(g)\right\|,\left\|\phi^{-M}(g)\right\|\right\}
$$


for all $g \in F$, where $\|$.$\| denotes the length of the conjugacy class of g$, $i$. e., the length of the shortest word in the conjugacy class of $g$.

Remark 2.2. The lemma shows that hyperbolicity is in fact a property of the outer automorphism represented by $\phi$, which will allow us to prove Theorem 1.1 by geometric methods. Lemma 2.3 rephrases the problem in terms of geometry.

Let $G$ be a finite graph with fundamental group $F$. We will always assume that a homotopy equivalence $f: G \rightarrow G$ maps vertices to vertices, and we will only consider graphs whose vertices have valence at least two. Following the conventions of [BFH98], we will refer to a map $\rho:[0,1] \rightarrow G$ as a path if it is either constant or an immersion, and we reserve the word circuit for immersions $\sigma: S^{1} \rightarrow G$.

We do not require paths to start or end at vertices. For a path or circuit $\rho$ in $G$, let $[f(\rho)]$ denote the path or circuit homotopic (relative end points if $\rho$ is a path) to the composition of $\rho$ with $f$. For a subpath $\rho$ of a path or circuit $\sigma$, let $\left[f^{k}(\rho)\right]_{\sigma}$ denote the maximal subpath of $\left[f^{k}(\rho)\right]$ contained in $\left[f^{k}(\sigma)\right]$.

Given a homotopy equivalence $f: G \rightarrow G$ of a graph and a path or circuit $\rho$ in $G$, we denote by $\rho^{-k}$ a path or circuit in $G$ with the property that $\left[f^{k}\left(\rho^{-k}\right)\right]=\rho$. Such a path $\rho^{-k}$ always exists, but it may not be unique if $\rho$ is not a circuit. However, all the statements we will prove will be independent of the choice of $\rho^{-k}$. Given some metric on $G$, we denote the length of $\rho$ by $L(\rho)$, and we restrict our attention to homotopy equivalences that map edges to paths of positive length.

Lemma 2.3. Let $f: G \rightarrow G$ be a homotopy equivalence representing an outer automorphism $\mathcal{O} \in$ Out $F$. If there exist numbers $M$ and $\lambda>1$ such that

$$
\lambda L(\sigma) \leq \max \left\{L\left(\left[f^{M}(\sigma)\right]\right), L\left(\sigma^{-M}\right)\right\}
$$

holds for all circuits $\sigma$ in $G$, then $\mathcal{O}$ is hyperbolic.

Proof. There exists a constant $C>0$ such that

$$
C^{-1} L(\sigma) \leq\|x\| \leq C L(\sigma)
$$

for all conjugacy classes $x$ in $F$ and circuits $\sigma$ in $G$ representing $x$. Choose $K$ such that $\lambda^{K}>C$. We conclude that

$$
\frac{\lambda^{K}}{C}\|x\| \leq \max \left\{\left\|\mathcal{O}^{K M}(x)\right\|,\left\|\mathcal{O}^{-K M}(x)\right\|\right\}
$$


for all conjugacy classes $x$.

In light of Lemma 2.3, we call a homotopy equivalence hyperbolic if it represents a hyperbolic automorphism.

\section{Train tracks}

In this section, we review the theory of train tracks developed in BH92, BFH98. We will restrict our attention to the collection of those results that will be used in this paper.

Oftentimes, a homotopy equivalence $f: G \rightarrow G$ will respect a filtration of $G$, i. e., there exist subgraphs $G_{0}=\emptyset \subset G_{1} \subset \cdots \subset G_{k}=G$ such that for each filtration element $G_{r}$, the restriction of $f$ to $G_{r}$ is a homotopy equivalence of $G_{r}$. The subgraph $H_{r}=\overline{G_{r} \backslash G_{r-1}}$ is called the $r$-th stratum of the filtration. We say that a path $\rho$ has nontrivial intersection with a stratum $H_{r}$ if $\rho$ crosses at least one edge in $H_{r}$.

If $E_{1}, \cdots, E_{m}$ is the collection of edges in some stratum $H_{r}$, the transition matrix of $H_{r}$ is the nonnegative $m \times m$-matrix $M_{r}$ whose $i j$-th entry is the number of times the $f$-image of $E_{j}$ crosses $E_{i}$, regardless of orientation. $M_{r}$ is said to be irreducible if for every tuple $1 \leq i, j \leq m$, there exists some exponent $n>0$ such that the $i j$-th entry of $M_{r}^{n}$ is nonzero. If $M_{r}$ is irreducible, then it has a maximal real eigenvalue $\lambda_{r} \geq 1$ (see [Sen73]). We call $\lambda_{r}$ the growth rate of $H_{r}$.

Given a homotopy equivalence $f: G \rightarrow G$, we can always find a filtration of $G$ such that each transition matrix is either a zero matrix or irreducible. A stratum $H_{r}$ in such a filtration is called zero stratum if $M_{r}=0 . H_{r}$ is called exponentially growing if $M_{r}$ is irreducible with $\lambda_{r}>1$, and it is called polynomially growing if $M_{r}$ is irreducible with $\lambda_{r}=1$.

An unordered pair of edges in $G$ originating from the same vertex is called a turn. A turn is called degenerate if the two edges are equal. We define a map $D f:\{$ turns in $G\} \rightarrow\{$ turns in $G\}$ by sending each edge in a turn to the first edge in its image under $f$. A turn is called illegal if its image under some iterate of $D f$ is degenerate, legal otherwise.

An edge path $\alpha=E_{1} E_{2} \cdots E_{s}$ is said to contain the turns $\left(\bar{E}_{i}, E_{i+1}\right)$ for $1 \leq i<s . \alpha$ is said to be legal if all its turns are legal, and a path $\alpha \subset G_{r}$ is $r$-legal if no illegal turn in $\alpha$ involves an edge in $H_{r}$.

A path $\rho$ in $G$ is said to be a (periodic) Nielsen path if $\rho$ is not constant and if $\left[f^{k}(\rho)\right]=\rho$ for some $k>0$. The period of $\rho$ is the smallest such 
exponent $k$. A pre-Nielsen path is a path whose image under some iterate of $f$ is a Nielsen path. A Nielsen path is called indivisible if it cannot be written as a concatenation of two Nielsen paths.

A decomposition of a path or circuit $\sigma=\sigma_{1} \cdots \sigma_{s}$ into subpaths is called a splitting if $\left[f^{k}\left(\sigma_{i}\right)\right]=\left[f^{k}\left(\sigma_{i}\right)\right]_{\sigma}$ for all $k, i$.

The following theorem was proved in BH92].

Theorem 3.1 (《BH92, Theorem 5.12]). Every outer automorphism $\mathcal{O}$ of $F$ is represented by a homotopy equivalence $f: G \rightarrow G$ such that each exponentially growing stratum $H_{r}$ has the following properties:

1. If $E$ is an edge in $H_{r}$, then the first and last edges in $f(E)$ are contained in $H_{r}$.

2. If $\beta$ is a nontrivial path in $G_{r-1}$ with endpoints in $G_{r-1} \cap H_{r}$, then $[f(\beta)]$ is nontrivial.

3. If $\rho$ is an r-legal path, then $[f(\rho)]$ is an r-legal path.

We call $f$ a relative train track map.

An outer automorphism $\mathcal{O}$ of $F$ is called reducible if it preserves the conjugacy class of a proper free factor of $F$. $\mathcal{O}$ is called irreducible if it is not reducible. If $\mathcal{O}$ is irreducible, then it has a relative train track representative $f: G \rightarrow G$ whose filtration has only one nonempty element $H_{1}=G$, with irreducible transition matrix. The properties of relative train tracks show that for every edge $E$ of $G$, the image $f^{n}(E)$ is an immersion for all $n>0$. In this case, we call $f$ a train track map (or absolute train track map), and we denote the growth rate of $H_{1}=G$ by $\lambda$.

We now construct a metric on $G$. If $H_{r}$ is an exponentially growing stratum, then its transition matrix $M_{r}$ has a unique positive left eigenvector $v_{r}$ (corresponding to $\lambda_{r}$ ) whose smallest entry equals one (see [Sen73]). For an edge $E_{i}$ in $H_{r}$, the eigenvector $v_{r}$ has an entry $l_{i}>0$ corresponding to $E_{i}$. We choose a metric on $G$ such that $E_{i}$ is isometric to an interval of length $l_{i}$, and such that edges in zero strata or in polynomially growing strata are isometric to an interval of length one. Note that if $\rho$ is a path whose endpoints are vertices, then the number of edges in $\rho$ provides a lower bound for $L(\rho)$. Moreover, if $f$ is an absolute train track map, then $f$ expands the length of legal paths by the factor $\lambda$. 
For our purposes, the properties of relative train track maps are not strong enough, so we will use the notion of improved train track maps constructed in [BFH98]. We only list the properties used in this paper.

Theorem 3.2 ([BFH98, Theorem 5.1.5]). For every outer automorphism $\mathcal{O}$ of $F$, there exists an exponent $k>0$ such that $\mathcal{O}^{k}$ is represented by a relative train track map $f: G \rightarrow G$ with the following additional properties:

1. Every periodic Nielsen path has period one.

2. $H_{r}$ is a zero stratum if and only if it is the union of the contractible components of $G_{r}$.

3. If $H_{r-1}$ is a zero stratum, then $H_{r}$ is an exponentially growing stratum.

4. If $H_{r}$ is a polynomially growing stratum, then $H_{r}$ consists of a single edge $E_{r}$, and $f\left(E_{r}\right)=E_{r} u_{r}$, where $u_{r} \subset G_{r-1}$.

5. If $H_{r}$ is an exponentially growing stratum, then there is at most one indivisible Nielsen path in $G_{r}$ that intersects $H_{r}$ nontrivially.

We call $f$ an improved relative train track map.

The following lemma is an immediate consequence of [BFH98, Lemma $5.1 .7]$.

Lemma 3.3. Suppose that $f: G \rightarrow G$ is an improved train track map representing an atoroidal automorphism, with an exponentially growing stratum $H_{r}$. If $\rho \subset G_{r}$ is a Nielsen path whose first and last edges are contained in $H_{r}$, then the endpoints of $\rho$ are distinct, and if both endpoints are contained in $G_{r-1}$, then at least one of them is contained in a contractible component of $G_{r-1}$.

The following lemma will turn out to be crucial in the proof of Theorem 1.1 .

Lemma 3.4. Let $f: G \rightarrow G$ be an improved train track map representing an atoroidal outer automorphism. If $H_{r}$ is an exponentially growing stratum and if $\rho$ is an indivisible Nielsen path in $G_{r}$ with nontrivial intersection with $H_{r}$, then the endpoints of $\rho$ are distinct and at least one of them is not contained in $H_{r} \cap G_{r-1}$. 
Proof. Since $\rho$ is indivisible, its initial edge and its terminal edge are contained in $H_{r}$. Suppose that both endpoints of $\rho$ are contained in $H_{r} \cap G_{r-1}$. By Lemma 3.3, one of them is contained in a contractible component of $G_{r-1}$. Let $v$ denote this endpoint. By Theorem 3.2, $H_{r-1}$ is necessarily a zero stratum, and we have $v \in H_{r-1}$. This implies that $f(v) \in G_{r-2}$. Since $H_{r-1}$ is the collection of contractible components of $G_{r-1}$, we conclude that $H_{r-1} \cap G_{r-2}=\emptyset$, which implies that $v \neq f(v)$. This contradicts our assumption that $\rho$ is a Nielsen path.

Finally, we state a lemma from BFH98 that simplifies the study of paths intersecting strata of polynomial growth. If $H_{r}=\left\{E_{r}\right\}$ is a polynomially growing stratum, then basic paths of height $r$ are of the form $E_{r} \gamma, \gamma \bar{E}_{r}$, or $E_{r} \gamma \bar{E}_{r}$, where $\gamma$ is a path in $G_{r-1}$ with endpoints in $H_{r}$.

Lemma 3.5 ([BFH98, lemma 4.1.4]). Let $f: G \rightarrow G$ be an improved train track map with a polynomially growing stratum $H_{r}$. If $\sigma$ is a circuit in $G_{r}$, then it splits as a concatenation of basic paths of height $r$ and paths in $G_{r-1}$.

Remark 3.6. In fact, part tof Theorem 3.2 implies that subdividing $\sigma$ at the initial endpoints of all occurrences of $E_{r}$ and at the terminal endpoints of all occurrences of $\bar{E}_{r}$ yields a splitting of $\sigma$ into basic paths of height $r$ and paths in $G_{r-1}$.

\section{Bounded cancellation}

Thurston's bounded cancellation lemma is one of the fundamental tools in this paper. We state it in terms of homotopy equivalences of graphs.

Lemma 4.1 (Bounded cancellation lemma, see [Co087]). Let $f: G \rightarrow$ $G$ be a homotopy equivalence. There exists a constant $C_{f}$, depending only on $f$, with the property that for any path $\rho$ in $G$ obtained by concatenating two paths $\alpha, \beta$, we have

$$
L([f(\rho)]) \geq L([f(\alpha)])+L([f(\beta)])-C_{f} .
$$


Bounded cancellation allows us to draw conclusions about the growth of sufficiently long paths under iterates of hyperbolic homotopy equivalences. We make this precise in the following lemma.

Lemma 4.2. Let $f: G \rightarrow G$ be a homotopy equivalence, and let $G^{\prime}$ be a subgraph of $G$ such that the restriction of $f$ to $G^{\prime}$ is a hyperbolic homotopy equivalence of $G^{\prime}$.

Then there exist constants $L^{C}, \lambda>1$ and $N$ with the property that, if $\rho \subset G^{\prime}$ is a subpath of some circuit $\sigma$ in $G$ and if the length of $\rho$ is at least $L^{C}$, we have

$$
\lambda L(\rho) \leq \max \left\{L\left(\left[f^{N}(\rho)\right]_{\sigma}\right), L(\alpha)\right\},
$$

where $\alpha$ is some subpath of $\sigma^{-N}$ satisfying $\left[f^{N}(\alpha)\right]_{\left(\sigma^{-N}\right)}=\rho$.

Proof. Let $g: G \rightarrow G$ be a homotopy inverse of $f$. Since the restriction of $f$ to $G^{\prime}$ is hyperbolic, there exist numbers $\lambda^{\prime}, N$ such that

$$
\lambda^{\prime} L\left(\sigma^{\prime}\right) \leq \max \left\{L\left(\left[f^{N}\left(\sigma^{\prime}\right)\right]\right), L\left(\left[g^{N}\left(\sigma^{\prime}\right)\right]\right)\right\}
$$

for all circuits $\sigma^{\prime}$ in $G^{\prime}$.

There exists a path $\tau \subset G^{\prime}$ such that $\sigma^{\prime}=[\rho \tau]$ is an immersed circuit in $G^{\prime}$, satisfying $L(\tau) \leq 2 \operatorname{diam}\left(G^{\prime}\right)$ and $L\left(\sigma^{\prime}\right) \geq L(\rho)-\operatorname{diam}\left(G^{\prime}\right)$. We can find some constant $K_{1}$, depending only on $G$ and $f$, such that $L\left(\left[g^{N}(\tau)\right]\right) \leq K_{1}$, $L\left(\left[f^{N}(\tau)\right]\right) \leq K_{1}$ and $\lambda^{\prime} \operatorname{diam}\left(G^{\prime}\right) \leq K_{1}$. We distinguish two cases that are not mutually exclusive.

1. $\lambda^{\prime} L\left(\sigma^{\prime}\right) \leq L\left(\left[f^{N}\left(\sigma^{\prime}\right)\right]\right)$. In this case, the bounded cancellation lemma tells us that

$$
\begin{aligned}
& \lambda^{\prime} L(\rho) \leq \lambda^{\prime}\left(L\left(\sigma^{\prime}\right)+\operatorname{diam}\left(G^{\prime}\right)\right) \leq L\left(\left[f^{N}\left(\sigma^{\prime}\right)\right]\right)+K_{1} \\
\leq & L\left(\left[f^{N}(\rho)\right]_{\sigma^{\prime}}\right)+2 K_{1} \leq L\left(\left[f^{N}(\rho)\right]_{\sigma}\right)+2 K_{1}+2 C_{f^{N}},
\end{aligned}
$$

where $C_{f^{N}}$ is the bounded cancellation constant of $f^{N}$.

2. $\lambda^{\prime} L\left(\sigma^{\prime}\right) \leq L\left(\left[g^{N}\left(\sigma^{\prime}\right)\right]\right)$. The same reasoning as in the previous case shows that $\lambda^{\prime} L(\rho) \leq L\left(\left[g^{N}(\rho)\right]_{\sigma}\right)+2 K_{1}+2 C_{g^{N}}$, where $C_{g^{N}}$ is the bounded cancellation constant of $g^{N}$. Since $f^{N}$ and $g^{N}$ are homotopy inverses of each other, we can find some constant $K_{2}$ such that $\left|L\left(\beta^{-N}\right)-L\left(\left[g^{N}(\beta)\right]\right)\right| \leq K_{2}$ holds for all paths $\beta$ and preimages $\beta^{-N}$ $\left(\beta^{-N}\right.$ is a preimage of $\beta$ under $\left.f^{N}\right)$. We conclude that

$$
\lambda^{\prime} L(\rho) \leq L(\alpha)+2 K_{1}+K_{2}+2 C_{g^{N}}
$$

where $\alpha$ is some subpath of $\sigma^{-N}$ satisfying $\left[f^{N}(\alpha)\right]_{\left(\sigma^{-N}\right)}=\rho$. 
Let $K=2 K_{1}+\max \left\{2 C_{f^{N}}, K_{2}+2 C_{g^{N}}\right\}$. Choose $L^{C}$ large enough to satisfy $\lambda^{\prime} L^{C}-K>L^{C}$ and let $\lambda=\frac{\lambda^{\prime} L^{C}-K}{L^{C}}$. Clearly, $\lambda>1$. If the length of $\rho$ is at least $L^{C}$, we conclude that

$$
\lambda L(\rho) \leq \lambda^{\prime} L(\rho)-K \leq \max \left\{L\left(\left[f^{N}(\rho)\right]_{\sigma}\right), L(\alpha)\right\} .
$$

We call $L^{C}$ the critical length of the triple $\left(f, G, G^{\prime}\right)$.

For train track maps, there is a related concept of critical length. Let $f: G \rightarrow G$ be a train track map with growth rate $\lambda$ and bounded cancellation constant $C_{f}$. If $\beta$ is a legal path in $G$ whose length satisfies $\lambda L(\beta)-2 C_{f}>$ $L(\beta)$ and $\alpha, \gamma$ are paths such that the concatenation $\alpha \beta \gamma$ is locally injective, then the length of the segment in $\left[f^{n}(\alpha \beta \gamma)\right]$ corresponding to $\beta$ will tend to infinity as $n$ tends to infinity. In this situation, the critical length is the infimum of the lengths satisfying the above inequality, i. e., $\frac{2 C_{f}}{\lambda-1}$.

\section{The irreducible case}

Throughout this section, let $f: G \rightarrow G$ denote a train track map representing an exponentially growing irreducible outer automorphism $\mathcal{O}$ of $F$, with growth rate $\lambda>1$. We equip $G$ with the metric constructed in Section 3 .

For a path or circuit $\rho$ in $G$, let $\mathcal{L}(\rho)$ denote the length of the longest legal segment of $\rho$ (recall that $L(\rho)$ denotes the length of $\rho$ ). Let $i(\rho)$ denote the number of illegal turns in $\rho$.

As in the previous section, we denote by $\rho^{-k}$ a path or circuit in $G$ with the property that $\left[f^{k}\left(\rho^{-k}\right)\right]=\rho$.

Let $C_{f}$ denote the bounded cancellation constant of $f$, and let $L^{c}=\frac{2 C_{f}}{\lambda-1}$ be the critical length of $f$.

We will use the following lemma from BFH97b].

Lemma 5.1. For all $L>0$ there exists an exponent $M>0$ such that if $\rho$ is any path in $G$, one of the following holds:

1. $\left[f^{M}(\rho)\right]$ has a legal segment of length greater than $L$.

2. $\left[f^{M}(\rho)\right]$ has fewer illegal turns than $\rho$. 
3. $\rho$ can be expressed as a concatenation $\tau_{1} \rho^{\prime} \tau_{2}$, where $L\left(\tau_{1}\right) \leq 2 L, L\left(\tau_{2}\right) \leq$ $2 L, i\left(\tau_{1}\right) \leq 1, i\left(\tau_{2}\right) \leq 1$, and $\rho^{\prime}$ splits as a concatenation of pre-Nielsen paths with one illegal turn each.

In order to study the length of preimages $\rho^{-k}$ of a path $\rho$, we will need an upper bound on $\mathcal{L}\left(\rho^{-k}\right)$ in terms of $\mathcal{L}(\rho)$. We make this precise in the following lemma.

Lemma 5.2. For all paths or circuits $\rho$ in $G$ and exponents $k>0$, we have

$$
\mathcal{L}\left(\rho^{-k}\right)<\frac{\mathcal{L}(\rho)}{\lambda^{k}}+L^{c} .
$$

In particular, this implies that $\mathcal{L}\left(\rho^{-k}\right)<\mathcal{L}(\rho)+L^{c}$.

Proof. We will show by induction that

$$
\mathcal{L}\left(\rho^{-k}\right) \leq \frac{\mathcal{L}(\rho)+2 C_{f}\left(1+\lambda+\cdots+\lambda^{k-1}\right)}{\lambda^{k}} .
$$

For $k=1$, the bounded cancellation lemma implies that

$$
\lambda \mathcal{L}\left(\rho^{-1}\right)-2 C_{f} \leq \mathcal{L}(\rho) \quad \Leftrightarrow \quad \mathcal{L}\left(\rho^{-1}\right) \leq \frac{\mathcal{L}(\rho)+2 C_{f}}{\lambda},
$$

so the claim holds for $k=1$.

Assume that the claim is true for some $k$. Again, the bounded cancellation lemma tells us that

$$
\mathcal{L}\left(\rho^{-(k+1)}\right) \leq \frac{\mathcal{L}\left(\rho^{-k}\right)+2 C_{f}}{\lambda} \leq \frac{\mathcal{L}(\rho)+2 C_{f}\left(1+\lambda+\cdots+\lambda^{k}\right)}{\lambda^{k+1}}
$$

by induction. Hence, we conclude that

$$
\mathcal{L}\left(\rho^{-k}\right) \leq \frac{\mathcal{L}(\rho)+2 C_{f} \frac{\lambda^{k}-1}{\lambda-1}}{\lambda^{k}}=\frac{\mathcal{L}(\rho)}{\lambda^{k}}+2 C_{f} \frac{1-\lambda^{-k}}{\lambda-1}<\frac{\mathcal{L}(\rho)}{\lambda^{k}}+\frac{2 C_{f}}{\lambda-1}
$$

While we measure the growth of paths under forward iteration by means of the path metric in $G$, our measure of growth under backward iteration will be the number of illegal turns. We make this precise in Lemmas 5.3 and 5.4 . Lemma 5.3 is a stronger version of [Lus98, Lemma 2]. 
Lemma 5.3. Let $f: G \rightarrow G$ be an improved train track map inducing an atoroidal outer automorphism $\mathcal{O}$. Given some number $L_{0}>0$, there exists some exponent $M>0$, depending only on $L_{0}$, such that for any path $\rho$ with $\mathcal{L}(\rho) \leq L_{0}$ and $i(\rho) \geq 4$, we have

$$
\left(\frac{8}{7}\right)^{n} i(\rho) \leq i\left(\rho^{-n M}\right)
$$

for all $n>0$.

Proof. Given $L_{0}$, choose an exponent $M$ according to Lemma 5.1, for $L=$ $L_{0}+L^{c}$. Express $\rho$ as a concatenation of paths $\rho_{1}, \cdots, \rho_{s}, \tau$ such that $i\left(\rho_{i}\right)=4$ and $i(\tau)<4$. There exist preimages $\rho_{1}^{-M}, \cdots, \rho_{s}^{-M}, \tau^{-M}$ such that $\rho^{-M}$ is their concatenation.

We claim that $i\left(\rho_{i}^{-M}\right) \geq 5$ for all $i$. Suppose otherwise, i. e., $i\left(\rho_{i}^{-M}\right)=4$ for some $i$. Because of our choice of $M$, Lemmas 5.1 and 5.2 imply that $\rho_{i}^{-M}$ can be written as a concatenation $\tau_{1} \rho^{\prime} \tau_{2}$, where $\rho^{\prime}$ splits as a concatenation of two pre-Nielsen paths with one illegal turn each. This implies that for some exponent $k \geq 0,\left[f^{k}\left(\rho^{\prime}\right)\right]$ contains the concatenation of two Nielsen paths, which is impossible because of Lemma 3.4 and Theorem 3.2, part 5 .

Hence, $i\left(\rho^{-M}\right) \geq 5 s+i(\tau) \geq\left(\frac{8}{7}\right) i(\rho)$, and the lemma follows by induction.

The following lemma establishes an elementary connection between the length of a path and the number of illegal turns.

Lemma 5.4. Given some $L>0$, there exists some constant $C>0$ such that for all paths $\rho$ with $1 \leq \mathcal{L}(\rho) \leq L$ and $i(\rho)>0$, we have

$$
C^{-1} i(\rho) \leq L(\rho) \leq C i(\rho) \text {. }
$$

A version of the following special case of Theorem 1.1 has already been proved in BFH97b. We present a new proof.

Theorem 5.5. If $f: G \rightarrow G$ is an improved train track map representing an irreducible, atoroidal outer automorphism of $F$, then $f$ is hyperbolic.

Proof. Fix some $L_{0}>L^{c}$.

Let $\sigma$ be a nontrivial circuit in $G$. We will distinguish several cases, and in each case we will show that there exist numbers $N>0, \lambda>1$ and $\epsilon>0$ such that there exists a collection $S$ of subpaths of $\sigma$ with the following properties: 
1. For every integer $n>0$ and for every $\rho \in S$, we have

$$
\lambda^{n} L(\rho) \leq \max \left\{\left[f^{n N}(\rho)\right]_{\sigma}, L(\alpha)\right\}
$$

where $\alpha$ is a subpath of $\sigma^{-n N}$ such that $\left[f^{n N}(\alpha)\right]_{\left(\sigma^{-n N}\right)}=\rho$. We say that $\rho$ has the desired growth.

2. There is no overlap between distinct paths in $S$.

3. The sum of the lengths of the paths in $S$ is at least $\epsilon L(\sigma)$.

If the numbers $N, \lambda$ and $\epsilon$ depend only on the case in question, but not on $\sigma$, then the theorem follows immediately because the growth of the subpaths in $S$ provides a lower bound for the growth of $\sigma$.

We distinguish the following cases.

1. $i(\sigma)=0$ or $\frac{L(\sigma)}{i(\sigma)} \geq L_{0}$. If $i(\sigma)=0$, then $\sigma$ is legal, so it has the desired growth under forward iteration. Otherwise, let $S$ be the collection of maximal legal subpaths of $\sigma$ of length at least $L_{0}$. The choice of $L_{0}$ and Lemma 4.1 guarantee that the subpaths in $S$ have the desired growth under forward iteration, so we only have to show that they account for a definite fraction of the length of $\sigma$. An elementary computation will verify this.

Let $l$ be the length of the longest path whose endpoints are vertices and whose length is strictly less than $L_{0}$. If $L(S)$ denotes the sum of the lengths of the segments in $S$, we have $L(\sigma)-L(S) \leq i(\sigma) l$ and $L(\sigma) \geq i(\sigma) L_{0}$. This implies that

$$
\frac{L(S)}{L(\sigma)}=1-\frac{L(\sigma)-L(S)}{L(\sigma)} \geq 1-\frac{l}{L_{0}}
$$

independently of $\sigma$.

2. $\frac{L(\sigma)}{i(\sigma)}<L_{0}$. There are two subcases to consider.

(a) $i(\sigma) \geq 4$. In this case, we define $S^{\prime}$ to be the set of subpaths left after removing from $\sigma$ the maximal legal segments of length greater than $6 L_{0}$. Then we obtain $S$ by removing from $S^{\prime}$ the subpaths with fewer than four illegal turns.

Lemma 5.3 and Lemma 5.4 (with $L=6 L_{0}$ ) show that the elements of $S$ have the desired growth under backward iteration, so we 
only have to show that $S$ accounts for a definite positive fraction of the length of $\sigma$. This fraction is minimal if $S$ contains only one subpath ( $S$ cannot be empty) and if all the paths in $S^{\prime} \backslash S$ have exactly three illegal turns. We first find a lower bound for the number of legal segments of the path in $S$ : Let $a$ be the number of legal segments of $\sigma$ of length greater than $6 L_{0}$, and let $b$ be the number of legal segments in $S$. Then $a \leq \frac{i(\sigma)}{6}$ and $i(\sigma)=b+a+4(a-1)$, which implies $b \geq \frac{i(\sigma)}{6}$. Moreover, the number of edges in a path provides a lower bound for the length of that path, so $b$ is also a lower bound for $L(S)$, and we conclude that

$$
\frac{L(S)}{L(\sigma)} \geq \frac{b}{L(\sigma)} \geq \frac{i(\sigma)}{6 L(\sigma)} \geq \frac{1}{6 L_{0}}
$$

(b) $i(\sigma)<4$. In this case, the length of $\sigma$ is bounded by $3 L_{0}$, so there are only finitely many circuits to consider. Since $\mathcal{O}$ is atoroidal, the length of all circuits tends to infinity under forward iteration, and we can easily find an exponent $N$ with the property that, say, $L\left(\left[f^{n}(\sigma)\right]\right) \geq 4 L_{0}$ for all circuits of length at most $3 L_{0}$ and for all $n \geq N$.

The cases considered above account for every circuit $\sigma$. This completes the proof.

\section{The reducible case - exponentially grow- ing strata}

Throughout this section, let $f: G \rightarrow G$ be a relative train track map representing an outer automorphism $\mathcal{O} \in$ Out $F$. The notation used in this section will be consistent with the notation in the previous section; the subscript $r$ will indicate the stratum of $G$ under consideration.

If $H_{r}$ is an exponentially growing stratum, let $\lambda_{r}$ be the corresponding growth rate. We equip $G$ with the metric constructed in Section 3 .

Let $\rho$ be a path or circuit in $G_{r}$. Following [BFH98], we denote by $\rho \cap H_{r}$ the ordered sequence of oriented edges of $H_{r}$ crossed by $\rho$. We will refer to the total length of $\rho \cap H_{r}$ as the $r$-length of $\rho$, denoted by $L_{r}(\rho)$. Similarly, $i_{r}(\rho)$ denotes the number of $r$-illegal turns in $\rho$, and $\mathcal{L}_{r}(\rho)$ stands for the 
$r$-length of the $\left(r\right.$-)longest $r$-legal segment of $\rho$. Let $L_{r}^{c}=\frac{2 C_{f}}{\lambda_{r}-1}$ be the critical $r$-length, where $C_{f}$ is the bounded cancellation constant of $f$. The relative train track property implies that $f$ expands the $r$-length of $r$-legal paths by the factor $\lambda_{r}$.

As in the previous section, we denote by $\rho^{-k}$ a path or circuit in $G$ with the property that $\left[f^{k}\left(\rho^{-k}\right)\right]=\rho$.

The following lemma is a straight-forward generalization of Lemma 5.2.

Lemma 6.1. Let $f: G \rightarrow G$ be a train track map with an exponentially growing stratum $H_{r}$, and let $\rho$ be a path or circuit in $G_{r}$. Then

$$
\mathcal{L}_{r}\left(\rho^{-k}\right)<\mathcal{L}_{r}(\rho)+L_{r}^{c}
$$

The following generalization of Lemma 5.1 is the main technical result of this section, and it will be crucial for our analysis of backward growth in the reducible case.

Proposition 6.2. Let $f: G \rightarrow G$ be a relative train track map, and let $H_{r}$ be an exponentially growing stratum. For all $L>0$, there exists some exponent $M>0$ such that if $\rho$ is a path in $G_{r}$ with $L_{r}(\rho) \geq 1$, one of the following three statements holds:

1. $\left[f^{M}(\rho)\right]$ has an $r$-legal segment of $r$-length greater than $L$.

2. $\left[f^{M}(\rho)\right]$ has fewer $r$-illegal turns than $\rho$.

3. $\rho$ can be expressed as a concatenation $\tau_{1} \rho^{\prime} \tau_{2}$, where $L_{r}\left(\tau_{1}\right) \leq 2 L$, $L_{r}\left(\tau_{2}\right) \leq 2 L, i_{r}\left(\tau_{1}\right) \leq 1, i_{r}\left(\tau_{2}\right) \leq 1$, and $\rho^{\prime}$ splits as a concatenation of pre-Nielsen paths (with one $r$-illegal turn each) and segments in $G_{r-1}$.

In order to prove Proposition 6.2, we will need the following version of a well-known fact from Ramsey theory.

Lemma 6.3. For all natural numbers $K, N_{0}, Q$ there exists some $M$ such that for all maps $f:\{1, \cdots, M\} \rightarrow\{1, \cdots, K\}$ there exist numbers $n$ and $N \geq N_{0}$ such that $f(n)=f(n+N)=\cdots=f(n+Q N)$. 
Proof. By GRS90, page 55, Theorem 2], there exists some number $M^{\prime}$ such that for all $f:\left\{1, \cdots, M^{\prime}\right\} \rightarrow\{1, \cdots, K\}$ there exist numbers $n$ and $N \geq 1$ such that $f(n)=f(n+N)=\cdots=f(n+Q N)$. Now $M=M^{\prime} N_{0}$ has the desired property.

Proof of Proposition 6.2. Fix some $N_{0}$ such that $\lambda_{r}^{N_{0}} \geq L$. Although there may be infinitely many paths $\rho^{\prime} \subset G_{r}$ whose endpoints are vertices and whose $r$-length is at most $3 L$, there is only a finite number $K$ of intersections $\rho^{\prime} \cap H_{r}$ of such paths with $H_{r}$.

Choose $M$ according to Lemma 6.3, with $Q=4$ and $K, N_{0}$ as above. We will show that $M$ is the desired exponent.

Let $\rho$ be a path in $G_{r}$ with $L_{r}(\rho) \geq 1$. Suppose that the first two statements do not hold for $\left[f^{M}(\rho)\right]$. We want to show that the third statement is satisfied. In order to avoid case distinctions, we assume that $i_{r}(\rho) \geq 4$; the proof in the case $i_{r}(\rho)<4$ is a straight-forward modification of the following argument.

Let $\rho_{1}^{k}, \cdots, \rho_{m}^{k}$ denote the $r$-legal segments of $\left[f^{k}(\rho)\right]$ for $k=0, \cdots, M$. By assumption, the $r$-length of each $\rho_{i}^{k}$ is bounded by $L$. Moreover, as the turn between two consecutive subpaths is $r$-illegal, the last edge in $\rho_{i}^{k}$ is contained in $H_{r}$ if $i<m$, and the first edge in $\rho_{i}^{k}$ is contained in $H_{r}$ if $i>1$.

Fix some $1<i<m-2$.2 By Lemma 6.3, there exist numbers $n$ and $N \geq N_{0}$ such that $\rho_{i}^{n} \cap H_{r}=\rho_{i}^{n+N} \cap H_{r}=\cdots=\rho_{i}^{n+4 N} \cap H_{r}, \rho_{i+1}^{n} \cap H_{r}=$ $\rho_{i+1}^{n+N} \cap H_{r}=\cdots=\rho_{i+1}^{n+4 N} \cap H_{r}$, and $\rho_{i+2}^{n} \cap H_{r}=\rho_{i+2}^{n+N} \cap H_{r}=\cdots=\rho_{i+2}^{n+4 N} \cap H_{r}$.

An elementary topological argument shows that there exist subpaths $\alpha^{\prime}$ of $\rho_{i}^{n} \rho_{i+1}^{n} \rho_{i+2}^{n}$ with the property $\alpha^{\prime} \cap H_{r}=\left[f^{N}\left(\alpha^{\prime}\right)\right] \cap H_{r}$ and $i_{r}\left(\alpha^{\prime}\right)=2$. Let $\alpha$ be the shortest such subpath. We will show that $\alpha$ can be expressed as a concatenation $\alpha_{1} \gamma \alpha_{2}$, where $\alpha_{1}, \alpha_{2}$ are pre-Nielsen paths, and $\gamma$ is a path that is constant or contained in $G_{r-1}$.

There exists a unique shortest subpath $\alpha_{1}$ of $\alpha$ such that $\alpha_{1}$ contains the first illegal turn of $\alpha$ and $\alpha_{1} \cap H_{r}=\left[f^{N}\left(\alpha_{1}\right)\right] \cap H_{r}$. Similarly, let $\alpha_{2}$ be the shortest subpath of $\alpha$ such that $\alpha_{2}$ contains the second illegal turn of $\alpha$ and $\alpha_{2} \cap H_{r}=\left[f^{N}\left(\alpha_{2}\right)\right] \cap H_{r}$. Note that the extremal (i.e., initial and terminal) edges of $\alpha_{1}, \alpha_{2}$ are (possibly partial) edges in $H_{r}$. We have $\alpha=\alpha_{1} \gamma \alpha_{2}$ for some path $\gamma \subset G_{r}$. If $\gamma$ were a path of positive $r$-length, this

\footnotetext{
${ }^{2}$ We do not consider the segments $\rho_{1}$ and $\rho_{m}$ in the following argument because the initial endpoint of $\rho_{1}$ (resp. the terminal endpoint of $\rho_{m}$ ) may not be a vertex. The paths $\tau_{1}$ and $\tau_{2}$ in the third statement account for $\rho_{1}$ and $\rho_{m}$.
} 
would imply that $L_{r}\left(f^{N}(\gamma)\right)>L_{r}(\gamma)$, contradicting our choice of $\alpha, \alpha_{1}$ and $\alpha_{2}$. We conclude that $\gamma$ is contained in $G_{r-1}$ or constant.

We claim that $\alpha_{1} \cap H_{r}=\left[f^{N}\left(\alpha_{1}\right)\right] \cap H_{r}=\cdots=\left[f^{4 N}\left(\alpha_{1}\right)\right] \cap H_{r}$. In order to see this, we need to understand the cancellation that occurs between the two maximal $r$-legal subpaths of $f^{N}\left(\alpha_{1}\right)$. In the tightening process, the terminal edge of the first subpath cancels with the initial edge of the second subpath until the last edge of the first subpath forms a nondegenerate turn with the first edge of the second subpath. Since $\left[f^{N}\left(\alpha_{1}\right)\right]$ contains an $r$-illegal turn, the resulting turn is necessarily $r$-illegal; in particular, it is contained in $H_{r}$.

This shows that the part of $f^{N}\left(\alpha_{1}\right)$ that is cancelled is completely determined by $\alpha_{1} \cap H_{r}$. Similarly, the part of $f^{N}\left(\left[f^{N}\left(\alpha_{1}\right)\right]\right)$ that is cancelled is completely determined by $\left[f^{N}\left(\alpha_{1}\right)\right] \cap H_{r}$, etc. Since $\alpha_{1} \cap H_{r}=\left[f^{N}\left(\alpha_{1}\right)\right] \cap H_{r}$, this shows that $\alpha_{1} \cap H_{r}=\left[f^{2 N}\left(\alpha_{1}\right)\right] \cap H_{r}$. We conclude that $\alpha_{1} \cap H_{r}=$ $\left[f^{N}\left(\alpha_{1}\right)\right] \cap H_{r}=\cdots=\left[f^{4 N}\left(\alpha_{1}\right)\right] \cap H_{r}$.

Let $E$ denote the first (possibly partial) edge of $\alpha_{1}$. The map $f^{N}$ expands the $r$-length of $E$ by $\lambda_{r}^{N}$, and it maps vertices to vertices, so $f^{N}(E)$ contains at least one entire edge in $H_{r}$, which implies that $f^{2 N}(E)$ has $r$-length at least $L$. The same argument applies to the last edge of $\alpha_{1}$, which implies that $\left[f^{2 N}\left(\alpha_{1}\right)\right]$ is completely determined by the extremal edges of $\alpha_{1}$. Applying this argument to $\left[f^{2 N}\left(\alpha_{1}\right)\right]$ and $\left[f^{4 N}\left(\alpha_{1}\right)\right]$, we conclude that $\left[f^{2 N}\left(\alpha_{1}\right)\right]=$ $\left[f^{4 N}\left(\alpha_{1}\right)\right]$, hence $\left[f^{2 N}(\alpha)\right]$ is a Nielsen path. The same argument shows that $\left[f^{2 N}\left(\alpha_{2}\right)\right]$ is a Nielsen path.

Repeating this argument for all indices $1<i<m-2$, we conclude that $\rho$ splits as a concatenation $\rho=\tau_{1} \beta_{1} \gamma_{1} \beta_{2} \gamma_{2} \cdots \beta_{m-3} \tau_{2}$, where $\tau_{1}$ and $\tau_{2}$ are as in the third statement, the paths $\beta_{i}$ are pre-Nielsen, and the paths $\gamma_{i}$ are contained in $G_{r-1}$ or constant. This completes the proof.

We will need the following relative versions of Lemmas 5.3 and 5.4 .

Lemma 6.4. Assume that $\mathcal{O}$ is atoroidal and that $f: G \rightarrow G$ is an improved train track representative with an exponentially growing stratum $H_{r}$. Given some number $L_{0}>0$, there exists some exponent $M>0$, depending only on $L_{0}$ and $H_{r}$, such that for any path $\rho$ in $G_{r}$ with $\mathcal{L}_{r}(\rho) \leq L_{0}$ and $i_{r}(\rho) \geq 5$, we have

$$
\left(\frac{10}{9}\right)^{n} i_{r}(\rho) \leq i_{r}\left(\rho^{-n M}\right)
$$

for all $n>0$. 
Proof. Given $L$, choose an exponent $M$ according to Lemma 6.2, with $L=$ $L_{0}+L_{r}^{c}$. Express $\rho$ as a concatenation of paths $\rho_{1}, \cdots, \rho_{s}, \tau$ such that $i_{r}\left(\rho_{i}\right)=$ 5 and $i_{r}(\tau)<5$. There exist preimages of $\rho_{1}^{-M}, \cdots, \rho_{s}^{-M}, \tau^{-M}$ such that $\rho^{-M}$ is their concatenation.

We claim that $i_{r}\left(\rho_{i}^{-M}\right) \geq 6$ for all $i$. Suppose otherwise, i. e., $i_{r}\left(\rho_{i}^{-M}\right)=5$ for some $i$. Because of our choice of $M$, Lemma 6.2 implies that $\rho_{i}^{-M}$ can be written as a concatenation $\tau_{1} \rho^{\prime} \tau_{2}$, and $\rho^{\prime}$ splits as a concatenation of three pre-Nielsen paths (with one illegal turn each) with (possibly empty) segments in $G_{r-1}$ in between. This implies that for some exponent $k \geq 0$, $\left[f^{k}\left(\rho^{\prime}\right)\right]$ contains three Nielsen paths with segments in $G_{r-1}$ in between, which is impossible because of Lemma 3.4 and Theorem 3.2, part 5 . tion.

Hence, $i_{r}\left(\rho^{-M}\right) \geq 6 s+i_{r}(\tau) \geq \frac{10}{9} i_{r}(\rho)$, and the lemma follows by induc-

Lemma 6.5. Suppose $H_{r}$ is an exponentially growing stratum. Given some $L>0$, there exists some constant $C>0$ such that for all paths $\rho \subset G_{r}$ with $1 \leq \mathcal{L}_{r}(\rho) \leq L$ and $i_{r}(\rho)>0$, we have

$$
C^{-1} i_{r}(\rho) \leq L_{r}(\rho) \leq C i_{r}(\rho)
$$

\section{Proof of the main theorem}

The following proposition and Lemma 2.3 immediately imply Theorem 1.1.

Proposition 7.1. If $f: G \rightarrow G$ is an improved relative train track map representing an atoroidal outer automorphism, then $f$ is hyperbolic.

Proof. We will proceed by induction up through the filtration of $G$ (as in the previous sections, we equip $G$ with the metric constructed in Section 3). The restriction of $f$ to $G_{1}=H_{1}$ is a homotopy equivalence, and we claim that $H_{1}$ is of exponential growth. If it were a zero stratum, this would imply that $f\left(H_{1}\right) \subset G_{0}$, but $G_{0}=\emptyset$. If it were of polynomial growth, it would give rise to a nontrivial fixed conjugacy class. We conclude that $H_{1}$ is of exponential growth, so this initial case follows from Theorem 5.5.

Now assume that the restriction of $f$ to $G_{r-1}$ is hyperbolic. We choose constants $L^{C}, \lambda$ and $N$ according to Lemma 4.2 for the triple $\left(f, G_{r}, G_{r-1}\right)$. 
As in the proof of Theorem 5.5, we will distinguish several cases, and in each case we will find a collection $S$ of subpaths having the desired growth and accounting for a definite positive fraction of the length of the circuit in question.

For the inductive step, we distinguish three main cases, depending on the stratum $H_{r}$.

1. $H_{r}$ is a zero stratum. Then $H_{r}$ is the collection of contractible components of $G_{r}$ (see Theorem 3.2). This implies that any nontrivial circuit in $G_{r}$ is contained in $G_{r-1}$, so there is nothing to show in this case.

2. $H_{r}$ is an exponentially growing stratum. We fix some length $L_{0}>$ $\max \left\{L^{C}, L_{r}^{c}\right\}$. Let $\sigma$ be a circuit in $G_{r}$ with nontrivial intersection with $H_{r}$. If $H_{r-1}$ is a zero stratum, we let $\sigma_{1}=\sigma \cap G_{r-2}, \sigma_{0}=\sigma \cap H_{r-1}$ and $\sigma_{2}=\sigma \cap H_{r}$. Otherwise, let $\sigma_{1}=\sigma \cap G_{r-1}, \sigma_{0}=\emptyset$ and $\sigma_{2}=\sigma \cap H_{r}$.

If $H_{r-1}$ is a zero stratum, then it is the collection of contractible components of $G_{r-1}$ (see Theorem 3.2). Consider a subpath $\rho$ of $\sigma$ that is contained in $H_{r-1}$. If $\rho$ is maximal, i. e., if $\rho$ is not a proper subpath of another subpath of $\sigma$ that is contained in $H_{r-1}$, then the edges preceding and following $\rho$ in the edge circuit $\sigma$ are contained in $H_{r}$.

This implies that $\frac{L\left(\sigma_{0}\right)}{L\left(\sigma_{2}\right)} \leq \operatorname{diam}(G)$ no matter whether $H_{r-1}$ is a zero stratum or not. Hence, $L(\sigma) \leq L\left(\sigma_{1}\right)+(1+\operatorname{diam}(G)) L\left(\sigma_{2}\right)$.

As in the proof of Theorem 5.5, we will decompose $\sigma$ into subpaths whose growth we understand. We consider several cases.

(a) $\frac{L\left(\sigma_{1}\right)}{L\left(\sigma_{2}\right)} \geq L_{0}$. In this case, there will be segments of length at least $L_{0}$ in $\sigma_{1}$, and the inductive hypothesis and Lemma 4.2 show that they have the desired growth. Hence, it suffices to show that those segments account for some definite fraction $\epsilon>0$ of the length of $\sigma$, where $\epsilon$ does not depend on the choice of $\sigma$. An elementary computation will verify this.

Let $A$ be the total length of all segments of length at least $L_{0}$ in $\sigma_{1}, B$ the total length of the remaining segments in $\sigma_{1}$, and let $C=L\left(\sigma_{2}\right)$. Then our assumption implies $A+B \geq L_{0} C$. Moreover, if $m$ denotes the number of segments in $\sigma_{1}$, we have $m \leq C$ and $\frac{A+B}{m} \geq \frac{L_{0} C}{m} \geq L_{0}$. 
We want to find a lower bound for $\frac{A}{L(\sigma)}$. Using the inequalities derived so far, we conclude that

$\frac{A}{L(\sigma)} \geq \frac{A}{A+B+(1+\operatorname{diam}(G)) C} \geq \frac{A L_{0}}{(A+B)\left(1+L_{0}+\operatorname{diam}(G)\right)}$

Hence, we only need to find a lower bound for $\frac{A}{A+B}$. Let $l$ be the length of the longest path whose endpoints are vertices and whose length is strictly less than $L_{0}$. Then $B \leq m l$ and $A+B \geq m L_{0}$, and we conclude that $\frac{A}{A+B}=1-\frac{B}{A+B} \geq 1-\frac{l}{L_{0}}$, independently of $\sigma$.

(b) $\frac{L\left(\sigma_{1}\right)}{L\left(\sigma_{2}\right)}<L_{0}$. In this case, significant growth will occur in $H_{r}$, and as in the proof of Theorem 5.5, we distinguish two subcases depending on whether forward or backward growth dominates.

i. $\frac{L_{r}(\sigma)}{i_{r}(\sigma)} \geq L_{0}$. In analogy with case $2 \mathrm{a}$, we only need to show that $r$-legal segments of $r$-length at least $L_{0}$ account for a definite fraction of the length of $\sigma$, which can be accomplished with a computation very similar to the one in case 2 a.

ii. $\frac{L_{r}(\sigma)}{i_{r}(\sigma)}<L_{0}$. As in the proof of Theorem 5.5, we consider two subcases.

A. $i_{r}(\sigma) \geq 5$. We define $S^{\prime}$ to be the set of subpaths left after removing from $\sigma$ the maximal $r$-legal subpaths of $r$-length greater than $7 L_{0}$, and we obtain $S$ from $S^{\prime}$ by removing subpaths with fewer than five $r$-illegal turns.

Lemma 6.1 (with $L=7 L_{0}$ ) and Lemmas 6.4 and 6.5 show that the paths in $S$ have the desired growth under backward iteration. An argument very similar to the one in the proof of Theorem 5.5 shows that the sum of the lengths of the paths in $S$ accounts for a definite positive fraction of the length of $\sigma$, so we are done in this case.

B. $i_{r}(\sigma)<5$. Only finitely many circuits $\sigma$ fall into this category, and the same argument as in the proof of Theorem 5.5 shows that they have the desired growth under forward iteration.

3. $H_{r}$ is a polynomially growing stratum. Recall (see Section 3) that $H_{r}$ contains only one edge $E_{r}$, and that basic paths of height $r$ are of the 
form $E_{r} \gamma, \gamma \bar{E}_{r}$, or $E_{r} \gamma \bar{E}_{r}$, where $\gamma$ is a path in $G_{r-1}$ with endpoints in $H_{r}$.

We fix some $L_{0}>L^{C}$. Let $\sigma$ be a circuit in $G_{r}$ with nontrivial intersection with $H_{r}$. Using Lemma 3.5 and Remark 3.6, we obtain a splitting of $\sigma$ by subdividing $\sigma$ at the initial endpoints of all occurrences of $E_{r}$ and at the terminal endpoints of all occurrences of $\bar{E}_{r}$. The subpaths of $\sigma$ obtained in this way are either basic paths of height $r$ or paths in $G_{r-1}$, and the endpoints of all subpaths are contained in $H_{r}$.

We first show that all basic paths of height $r$ have the desired growth under sufficiently high iterates of $f^{ \pm 1}$. Let $\rho$ be a basic path of height $r$. Since a basic path of the form $\gamma \bar{E}_{r}$ can be turned into a basic path of the form $E_{r} \gamma$ by reversing its orientation, we only have to distinguish two cases.

(a) $\rho=E_{r} \gamma \bar{E}_{r}$ with $\gamma \subset G_{r-1}$. If $L(\gamma) \geq L_{0}$, the inductive hypothesis and Lemma 4.2 prove the claim, so it suffices to consider the case $L(\gamma)<L_{0}$. The endpoints of $\gamma$ are equal, and we denote by $\tau$ the circuit defined by $\gamma$. In general, $\tau$ may be shorter than $\gamma$ because initial and terminal edges of $\gamma$ may cancel. However, we have $L(\tau) \geq 1$ and $L(\gamma)-L(\tau)<L_{0}$. Moreover, the growth of $\tau$ under iterates of $f$ provides a lower bound for the growth of $\rho$ under iterates of $f$, so the inductive hypothesis proves the claim in this case.

(b) $\rho=E_{r} \gamma$ with $\gamma \subset G_{r-1}$. As in the previous case, we may assume that $L(\gamma)<L_{0}$. We first show that $[f(\rho)] \neq \rho$. Suppose otherwise. Then the endpoints of $\rho$ cannot be equal because $\mathcal{O}$ is atoroidal. This implies that the endpoints of $E_{r}$ are distinct and $\gamma$ starts and ends at the terminal endpoint of $E_{r}$. However, this is impossible as it implies that $\rho$ is a basic path of the form $E_{r} \gamma \bar{E}_{r}$ (see Lemma 3.5 and Remark 3.6).

We conclude that $\lim _{n \rightarrow \infty} L\left(\left[f^{n}(\rho)\right]\right)=\infty$. As there are only finitely many paths of length less than $L_{0}$, we conclude that the circuits in this category have the desired growth under forward iteration.

We have shown that basic paths of height $r$ have the desired growth, as do paths in $G_{r-1}$ if their length is at least $L_{0}$. This leaves us with those 
subpaths in the splitting of $\sigma$ that are contained in $G_{r-1}$ and whose length is less than $L_{0}$, but we can safely disregard them because there are at least as many basic paths of height $r$ as there are subpaths in $G_{r-1}$. This completes the proof.

\section{References}

[BF92] M. Bestvina and M. Feighn. A combination theorem for negatively curved groups. J. Diff. Geom., 35:85-101, 1992.

[BF96] M. Bestvina and M. Feighn. Addendum and correction to: "A combination theorem for negatively curved groups". J. Diff. Geom., 43:783-788, 1996.

[BFH97a] M. Bestvina, M. Feighn, and M. Handel. Erratum to: "Laminations, trees, and irreducible automorphisms of free groups". Geom. Funct. Anal., 7(6):1143, 1997.

[BFH97b] M. Bestvina, M. Feighn, and M. Handel. Laminations, trees, and irreducible automorphisms of free groups. Geom. Funct. Anal., 7 (2):215-244, 1997.

[BFH98] M. Bestvina, M. Feighn, and M. Handel. The Tits alternative for $\operatorname{Out}\left(F_{n}\right)$ I: Dynamics of exponentially growing automorphisms. preprint, 1998.

[BH92] M. Bestvina and M. Handel. Train tracks and automorphisms of free groups. Ann. of Math., 135:1-51, 1992.

[Coo87] D. Cooper. Automorphisms of free groups have finitely generated fixed point sets. J. Alg., 111:453 - 456, 1987.

[Ger98] S.M. Gersten. Cohomological lower bounds for isoperimetric functions on groups. Topology, 37:1031-1072, 1998.

[GRS90] R. Graham, B. Rothschild, and J. Spencer. Ramsey theory. WileyInterscience, 1990. 
[Lus98] M. Lustig. Discrete actions on the product of two non-simplicial R-trees. preprint, 1998.

[Sen73] E. Seneta. Non-Negative Matrices. John Wiley \& Sons, 1973.

Department of Mathematics, University of Utah Salt Lake City, UT 84112, USA

E-mail: brinkman@math.utah.edu 